\title{
Control Charts Limits Flexibility Based on the Equipment Conditions
}

\author{
S. Lampreia, V. Vairinhos, V. Lobo \& R. Parreira
}

1, vlobo@isegi.unl.pt, ribeiro.parreira@marinha.pt, Centro de Investigação Naval (CINAV), Alfeite, 2810 Almada, Portugal

\section{J. G. Requeijo}

ifgr@fct.unl.pt, Faculty of Science and Technology of the Universidade Nova of Lisbon, Mechanical and Industrial Engineer Department, Lisbon. 2829-516, Caparica, Portugal

\begin{abstract}
Condition Based Maintenance became an important development in industrial and transport equipment maintenance efforts. Many statistical methodologies have been applied in this area. These methodologies are usually applied off-line: after the data is collected. We propose an online, real-time condition monitoring system based on a modified control chart, applied to engine parameters. These charts should be flexible enough and its control limits should reflect the equipment state, the manufacturer specifications and onboard meteorological conditions. In this study we will develop a methodology to specify flexible chart control limits. The experimental equipment is a combined diesel or gas propulsion system. Two phases will be assumed. In phase 1 the equipment and historical data are analyzed, studying historical data, which leads to the definition of equipment parameters. In phase 2, new data is obtained by simulation, and the Exponentially Weighted Moving Average charts are applied considering flexible limits.
\end{abstract}

\section{INTRODUCTION}

\subsection{Applying control charts}

Control charts may be used in conjunction with Remaining Useful Life (RUL) - as an indicator of equipment condition - to support maintenance decision making. See Rawat (2013). Both traditional or Shewart charts assume two phases. In phase 1, data parameters are defined and estimated, and on phase 2 the use of special control charts can enhance detection sensitivity. Applying Statistic Process Control (SPC) to equipment -Statistical Equipment Control (SEC) - it is possible to detect out of control events (Lampreia, 2013)

\subsection{The importance of limits flexibility}

Flexible control chart limits allow charts to adapt (Schaeffers, 2016) to ships new operational areas. For example: the operation of a propulsion diesel engine in areas with sea water temperature below $14^{\circ} \mathrm{C}$ is not the same as the operation in areas with temperatures in the interval $15-25^{\circ} \mathrm{C}$ or above $25^{\circ} \mathrm{C}$. But even this flexibility should have limits. These limits are the ones imposed by the manufacturer.

\section{CONTROL CHARTS}

\subsection{Phase 1 - Traditional Control Charts}

Having enough data to estimate the process parameters, phase 1 of SPC with Shewhart charts is implemented. The volume of data (number of samples $(m))$ needed to define the parameters on phase 1 depends on the sample dimension $(n)$. Queensberry see (Pereira \& Requeijo, 2012) - suggests, as can be seen in equation 1 , that:

$m \geq \frac{400}{(n-1)}$

For individual For a single sample, the same author suggests that 300 observations should be used. Lampreia (2013), using experimental equipment data uses 200 observations on phase. In the present work it is shown that 200 observations are enough.

In this article individual observation (X) and moving range (MR) control charts are implemented considering continuous and independent data. The control charts analysis must show controlled statistical equipment.

For independent data, phase 1 should proceed and the Shewhart or traditional control charts are built. Upper control limit (UCL), Lower control limit (LCL) and Center line (CL) for those charts, are calculated using the formulas on the table 1.These cal- 
culations, based on the $\mathrm{m}$ (or $m-1$ ) sample dimensions are the statistics $\bar{X}=\sum_{i=1}^{m} X_{i} / m \quad$ and $\overline{M R}=\sum_{i=1}^{m-1} M R_{i} /(m-1)$, where $M R_{i}=\left|X_{i}-X_{i-1}\right|$.

Table 1. Traditional Control Charts Limits, in phase 1 of SPE (Pereira e Requeijo, 2012)

\begin{tabular}{|c|c|c|c|}
\hline Chart & $L C L$ & $C L$ & $U C L$ \\
\hline$X$ & $\bar{X}-3 \sigma_{X}$ & $\bar{X}$ & $\bar{X}+3 \sigma_{X}$ \\
\hline $\begin{array}{c}M R \text { (moving } \\
\text { range) }\end{array}$ & $D_{3} \overline{M R}$ & $\overline{M R}$ & $D_{4} \overline{M R}$ \\
\hline
\end{tabular}

If the process is stabilized - under statistical control -its parameters should be estimated by $\hat{\mu}=\bar{X}$ and $\hat{\sigma}=\overline{M R} / d_{2}$. The constants $D_{3}, D_{4}$ and $d_{2}$ depend exclusively on the sample dimension. (Lampreia et al, 2012)

For auto correlated/dependent data, since we are working in condition based maintenance, a fitted statistical model for the autocorrelation should be applied. We suggest the ARIMA (Autoregressive Integrated Moving Average) (p, d, q) Model. The statistics from individual observation and Moving Range control charts are calculated using the fitted model residues for every observation. (Lampreia et al, 2013).

Estimated -sampling - Autocorrelation Function (ACF) and Partial Auto-Correlation Function (PACF) are compared with (ACF) and (PACF) of known dependence patterns in order to choose a specific model.

Equipment parameters follows a $\operatorname{ARIMA~}(p, d, q)$ model if $\nabla^{d} X_{t}$. The model defined by ARIMA $(\mathrm{p}, \mathrm{d}, \mathrm{q})$ :

$$
\begin{aligned}
& \Phi_{p}(B) \nabla^{d}=X_{t}=\Theta_{q}(B) \varepsilon_{t} \\
& \Phi_{p}(B)=\left(1-\phi_{1} B-\phi_{2} B^{2}-\ldots-\phi_{p} B^{p}\right) \\
& \Theta_{q}(B)=\left(1-\theta_{1} B-\theta_{2} B^{2}-\ldots-\theta_{q} B^{q}\right) \\
& B=\frac{X_{t-1}}{X_{t}} \mathrm{e} \nabla=\frac{X_{t}-X_{t-1}}{X_{t}} \\
& E(X)=\mu=\frac{\xi}{1-\sum_{j=1}^{p} \phi_{j}} \\
& E(X)=\mu
\end{aligned}
$$

Where $B$ is the lag operator, $\nabla$ is the difference operator, $d$ is the differentiation order to render a stationary process, $X_{t}$ is the observation at time $t, \varepsilon_{t}$ is the white noise at time $t, \Phi(B)$ is the autoregressive polynomial of order $p$ and $\Theta(B)$ is the moving average polynomial of order $q$.
Using a defined model, the residues are estimated by $e_{t}=X_{t}-\hat{X}_{t}$, with $\hat{X}_{t}$ expected value for the period $t$.

For more details Pereira and Requeijo (2012) should be consulted.

\subsection{Phase 2 - Modified EWMA Chart}

In phase 2, an EWMA control chart becomes suitable for equipment monitoring if the process has a change; $T_{L}$ represent the limit imposed by the manufacturer.

The variable that defines the Modified EWMA is the exponentially weighted variable, $\mathrm{E}$, defined by the equation (2) (Crowder \& Hamilton, 1992).

$E_{t}=\max \left(0,(1-\lambda) \mathrm{E}_{t-1}+\lambda\left(\bar{X}_{t}-T_{L}\right)\right), 0<\lambda>1$

where, $\sigma_{\bar{X}}=\sigma_{\varepsilon} / \sqrt{n}, \Delta=\delta \sigma_{\bar{X}}$, (Zou \& Tsung, 2010) $T_{L}=\left(T_{L}\right)_{S \operatorname{tandard}}-\Delta_{S}$ and $\Delta_{S}=\delta_{1} \sigma$, with $\delta_{1}$ as a constant.

In these equations $\bar{X}_{t}$ is the sample mean at $\mathrm{t}$ (Serel \& Moskowitz, 2008), $\mathrm{T}_{\mathrm{L}}$ the maximum or minimum admissible for each variable or parameter, $\sigma_{E}$ the variable $\mathrm{E}$ standard deviation, $\mathrm{n}$ the sample dimension, $\lambda$ the weight constant $(0 \leq \lambda \leq 1)$ and $\Delta_{\mathrm{s}}$ the safety factor. The Modified EWMA limits are Alert Level (AL) and Upper Control Level (UCL) for these charts are given by equations (3) and (4). (Lampreia et al, 2012)

$$
\begin{aligned}
& A L_{E}=K_{1} \sigma_{E} \\
& U C L_{E}=K_{2} \sigma_{E}
\end{aligned}
$$

where

$$
\sigma_{E}=\sigma_{\bar{x}} \sqrt{\frac{\lambda}{2-\lambda}}
$$

$\mathrm{K}_{1}$ and $\mathrm{K}_{2}$ are functions of values of $\lambda$ and ARL (for AL and UCL). To define the AL and UCL Crowder (1989) abacus are used, entering with ARL (Average Run Length) value and the mean change, $\delta$, where we obtain $\mathrm{K}_{1}$ and $\mathrm{K}_{2}$. In this study $\alpha=1 \%$ $(A R L=100)$ will be considered to define $\mathrm{AL}$ and $\alpha=0,2 \%(A R L=500)$ to define UCL.

\section{METHODOLOGY}

Depending on equipment characteristics, we proposed a methodology to be applied to repairable systems monitoring.

For independent data the steps should be as follows:

- Collect data. At least 200 observations. 
- With the equipment in good performance check for data independence and Normality. Estimate its parameters;

- For online equipment monitoring, build the Modified EWMA charts using the online collected values.

- Define the change in the parameters mean that must be detected.

- Estimate $A L$ and $U C L$ control limits, considering some meteorological standards.

- Fix the intervention rules:

- 6 consecutive points are above the $A L$ Proceed to an inspection.

- 3 consecutive points are above the $U C L$ - Proceed to a maintenance intervention.

\section{RESULTS}

\subsection{Phase 1-Traditional Control Charts}

The variables under study are from a Combined Diesel or Gas propulsion system associated to a pitch propeller (PP). See Table 2 for the meaning of variables. Between 0 to 500 of engine rpm PP is variable; after that value, for more speed, only the rpm of the engine is increased.

Phase 1 starts with the study of 200 observations collected from four parameters of an engine, at constant power. This study includes normality and independence checks and parameters definition.

Table 2. Variables in Study

\begin{tabular}{|c|c|}
\hline OP0155 & Lub Oil Pressure \\
\hline OP0157 & Piston Cooling Oil Pressure \\
\hline FP0163 & Fuel Oil Pressure \\
\hline OT0114 & Lub Oil Temperature \\
\hline
\end{tabular}

The Normality and the independence study explained in this article is for variable number 1 .

For variable 1, normality was tested with the Kolmogorov-Smirnov test. See Figure 1, Figure 1, where $D_{\text {Critico }}=\frac{0,886}{\sqrt{N}}=\frac{0,886}{\sqrt{200}}=0,06265$ for $\quad \alpha=5 \%$, and $\mathrm{d}=0,03627$. Once $d<D_{\text {Critico }}$ the normality condition is accepted. (Pereira e Requeijo, 2012)



Figure 1. Var1 - Normality Study

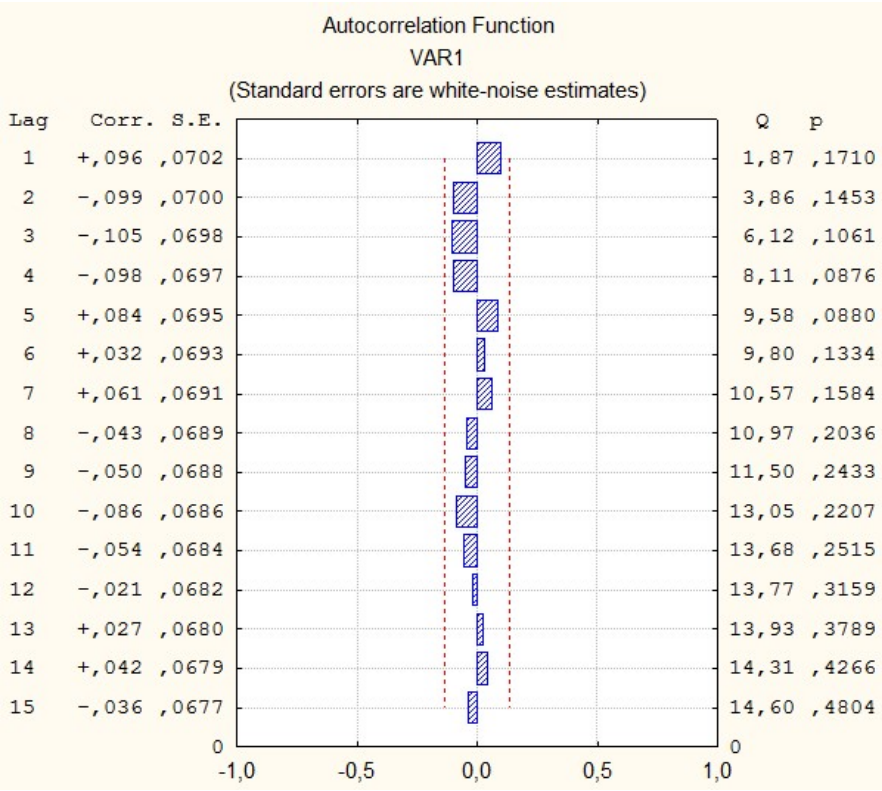

Figure 2. Var1 - ACF

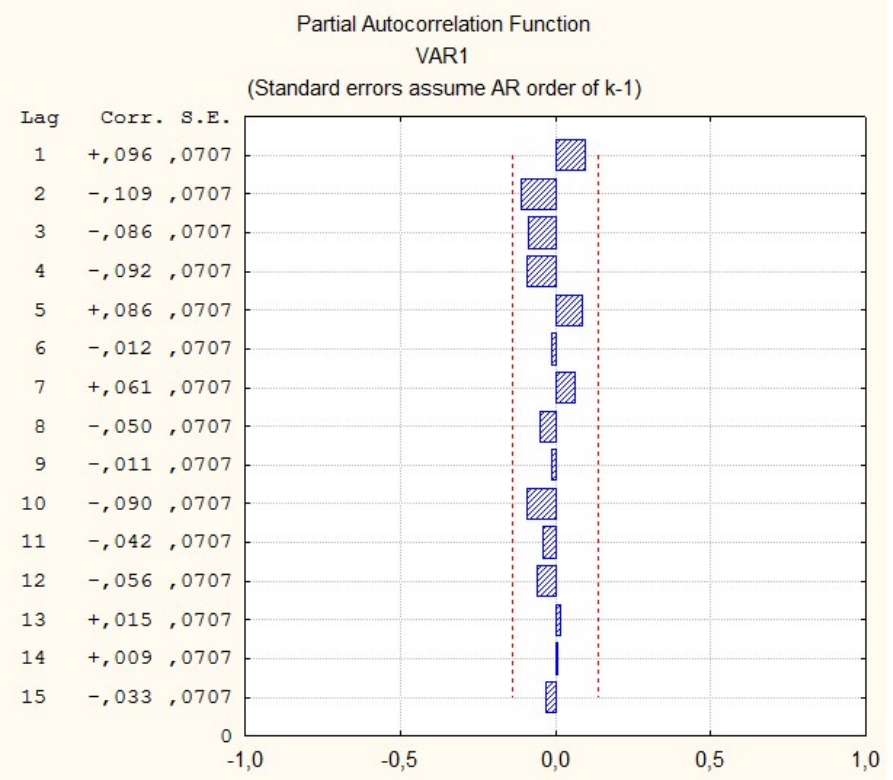

Figure 3. Var1 - PACF

All the variables were found to be normal and independent. 
When building traditional control charts some outliers were found. To estimate the parameters, outliers must be excluded or replaced, using some criteria; our decision was its replacement with the mean between the preceding and the next values.

Using Statistica software, the following results were obtained before replacing outliers:

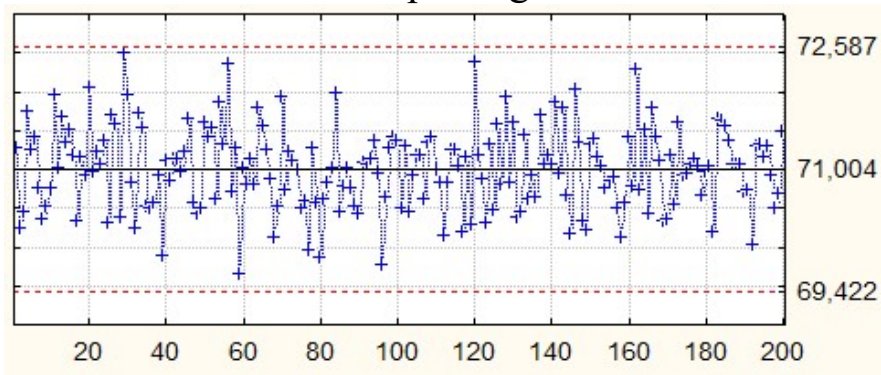

Figure 4. Var 4 (Lub Oil Temperature) - Individual Control Chart - Without Review

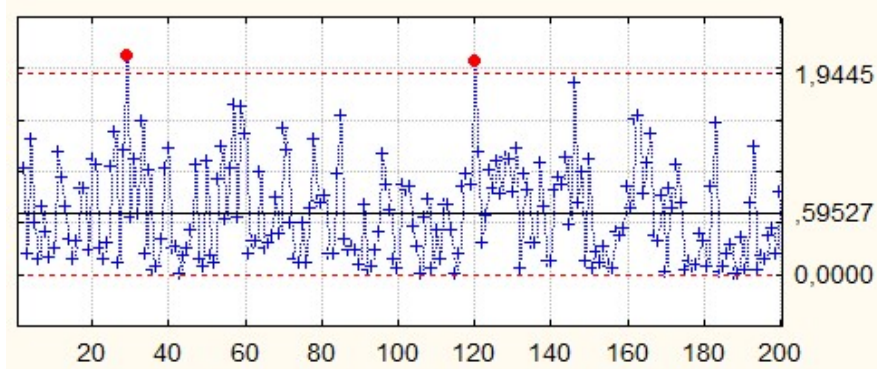

Figure 5. Var 4 (Lub Oil Temperature)- Moving Range Control Chart - Without Review

The results after replacing outliers:

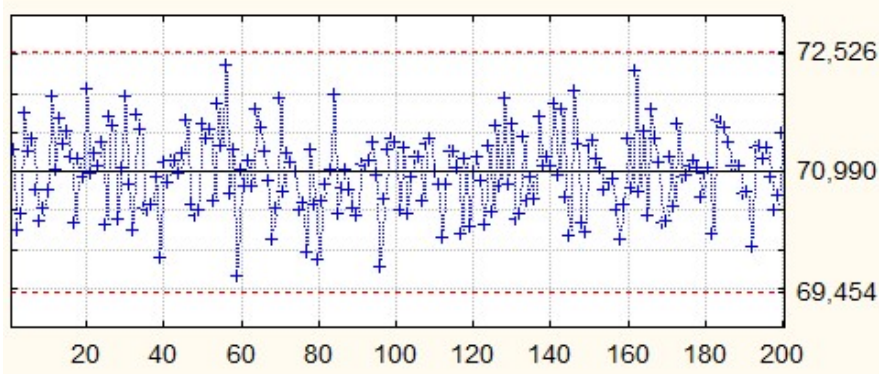

Figure 6. Var 4 (Lub Oil Temperature) - Individual Control Chart - Reviewed

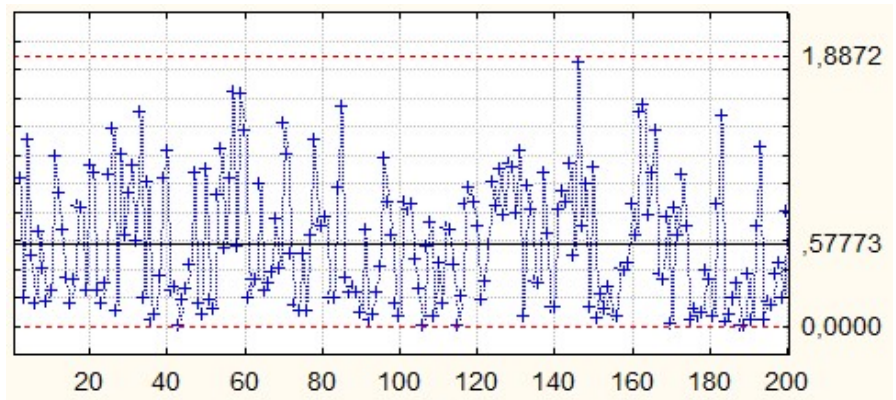

Figure 7. Figure 4. Moving Range Var 4 (Lub Oil Temperature) - Reviewed

Applying the same procedure to others variables we obtained the parameters estimations for $\mu$ and $\sigma$. See results on Table 3., obtained again with Statistica.
Table 3. Variables parameters

\begin{tabular}{|c|c|c|c|c|}
\cline { 2 - 5 } \multicolumn{1}{c|}{} & VAR 1 & VAR 2 & VAR 3 & VAR 4 \\
\hline$\mu$ & 6,3529 & 5,9947 & 0,79116 & 70,99 \\
\hline$\sigma$ & 0,0009 & 0,06856 & 0,3778 & 0,512 \\
\hline$T_{L}$ & 5,5 & 5,5 & 0,5 & 95 \\
\hline
\end{tabular}

\subsection{Phase 2 - Modified EWMA Control Charts Lim- its Flexibility}

To sum up: we observed that for some operating conditions, the ship diesel propulsion parameters show considerable variability, so we will consider 3 limits according sea temperature, at a cruise speed with maximum pitch propeller and 800 engine rpm. In what follows, specifically for the limits flexibility we present Var 4 (Lub oil temperature) study in more detail, with 3 distinct $\mathrm{T}_{\mathrm{L}}$ values ( lower, normal and higher namely 80,85 and $95^{\circ} \mathrm{C}$ ). For this specific parameter, the ship sensor program has an alarm.

It is considered important to detect eventual damage on the selected variables with $\sigma$ three levels of variation. To test its sensibility, $\Delta=[0,5 \sigma ; 1 \sigma ; 1,5 \sigma]$, $\delta=[0,5 ; 1 ; 1,5]$, with $\alpha=1 \%(\mathrm{ARL}=100)$ in the definition of $\mathrm{AL}$ and $\alpha=0.2 \%(\mathrm{ARL}=500)$ in the definition of UCL. Using the Crowder abacus (Pereira and Requeijo, 2012), we obtained $\lambda=0.05, \lambda=0,13$ and $\lambda=0.25$, respectively, and $K=2.7, K=2.9$ and $K=3$.

AL and the UCL values for each variable and each $\sigma$ are obtained using expressions 2 and 3 ,

Because this is a real engine, no induced damage is allowed, so the parameters with anomalies were simulated in the MATLAB, considering 0 to 3 progressions of the anomalies of the engine, and considering the limits flexibility

For sea mean temperature, with $T_{L}=85^{\circ} \mathrm{C}$, for the $2^{\text {rd }}$ progression of a possible anomaly, considering $\alpha=1 \% \quad(A R L=100) \mathrm{AL}$ and $\alpha=0,2 \% \quad(A R L=500)$ to UCL.

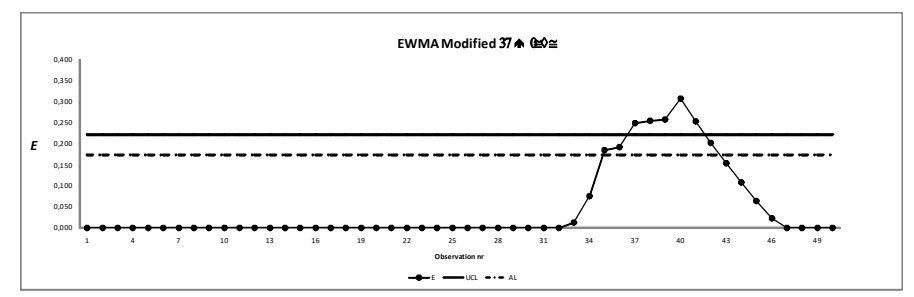

Figure 8. Var4 $-T_{L}=85^{\circ} \mathrm{C}$ with $\Delta=0,5 \sigma-2^{\text {a Progression }}$ 


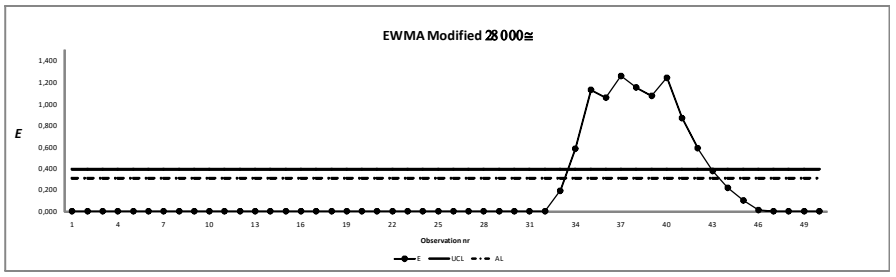

Figure 9. Var4 $-T_{L}=85^{\circ} \mathrm{C}$ with $\Delta=1 \sigma-2^{\text {a Progression }}$

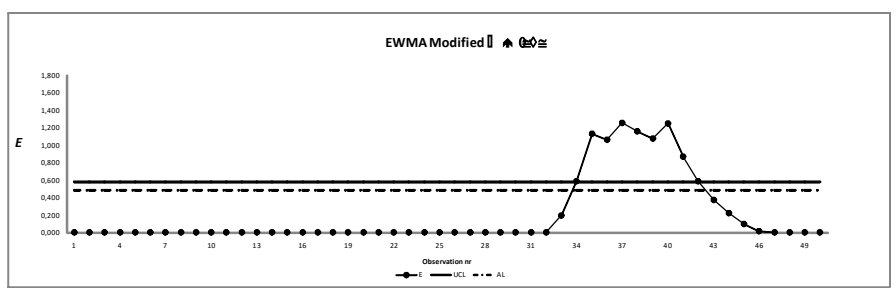

Figure 10 . $\operatorname{Var} 4-T_{L}=85^{\circ} \mathrm{C}$ with $\Delta=1,5 \sigma-2^{\text {a Progression }}$

Comparing the results for each $\Delta$, we fixed $\Delta=0,5 \sigma$ as the more suitable for the variables characteristics and results. Analyzing figure 4, and using the defined methodology, we should proceed to an investigation action by the $40^{\text {th }}$ observation.

With $T_{L}=85^{\circ} \mathrm{C}$, for the $3^{\text {rd }}$ progression of a possible anomaly, figure 11:

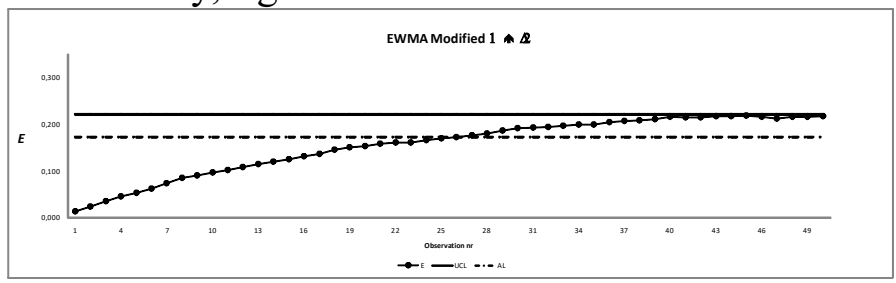

Figure 11. Var4 $-T_{L}=85^{\circ} \mathrm{C}$ with $\Delta=1,5 \sigma-3^{\text {a Progression }}$

In figure 11 the chart shows that engine needs an investigation action after 31 th observation.

Figure 12 shows an intervention need after 14th observation.

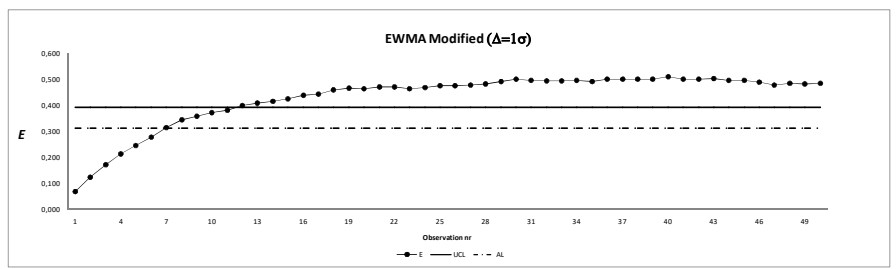

Figure 12. Var4 $-T_{L}=85^{\circ} \mathrm{C}$ with $\Delta=1 \sigma-3^{\text {aProgression }}$

If we consider $T_{L}=80^{\circ} \mathrm{C}$, all the observations will be out of control; see figure 13. If we assume $T_{L}=95^{\circ} \mathrm{C}$ all the observations are under control. See figure 14.

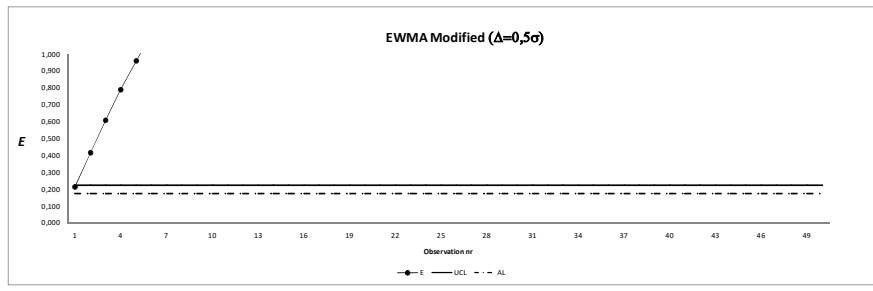

Figure 13. $\operatorname{Var} 4-T_{L}=80^{\circ} \mathrm{C}$ with $\Delta=0,5 \sigma-3^{\text {aPProgression }}$

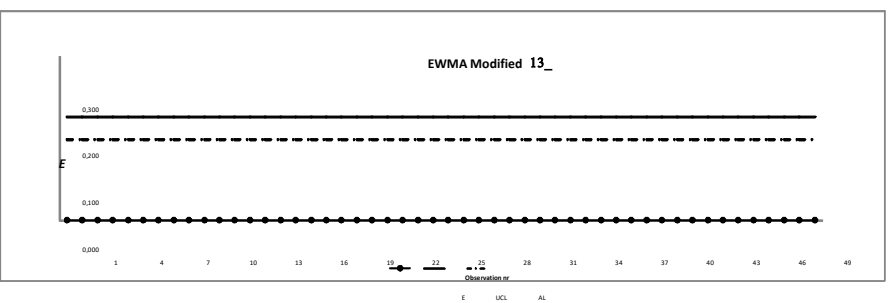

Figure 14. Var4 $-T_{L}=95^{\circ} \mathrm{C}$ with $\Delta=0,5 \sigma-3^{\text {aPProgression }}$

This means that, with the variation on the $\mathrm{T}_{\mathrm{L}}$ value, the results change. If $T_{L}$ decreases, the results decreases; with a $T_{L}$ increase there is a rise in the resulting values.

To change the limits, the assumed ARL and $\delta$ should change. We simulated several values, considering only $\delta$ with $0.5,1$, and 1.5 , given the Crowder abacus design.

Considering $\quad \alpha=1 \% \quad(A R L=100) \quad A L \quad$ and $\alpha=0,2 \%(A R L=2000)$ to $U C L$, figure 15 and 16 :

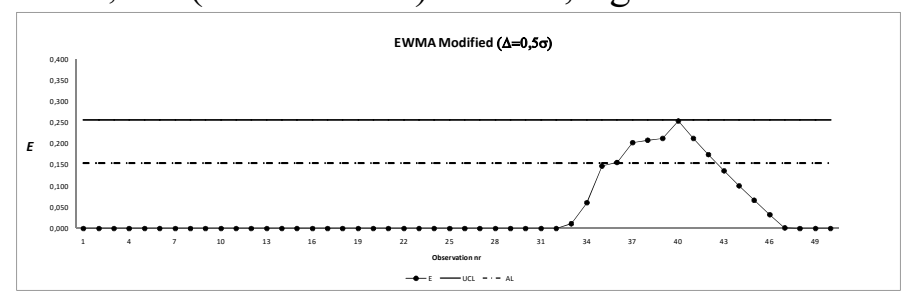

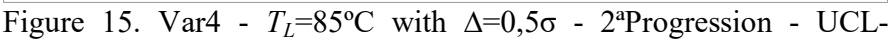
$\mathrm{ARL}=2000$

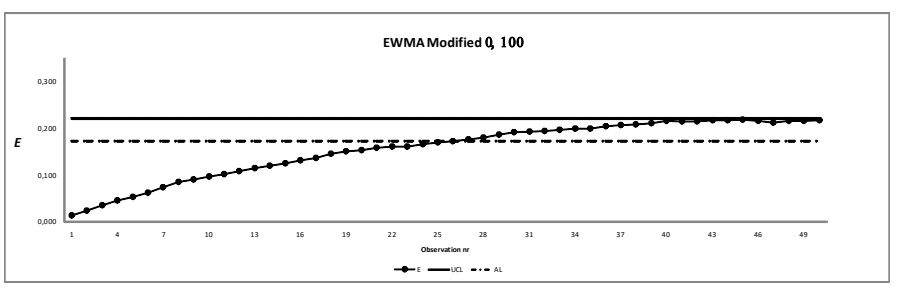

Figure 16. Var4 - $T_{L}=85^{\circ} \mathrm{C}$ with $\Delta=0,5 \sigma-3^{\text {aaProgression - UCL- }}$ $\mathrm{ARL}=2000$

Considering $\quad \alpha=1 \% \quad(A R L=100) \quad \mathrm{AL} \quad$ and $\alpha=0,2 \%(A R L=1000)$ to $U C L$, figure 17 and 18 :

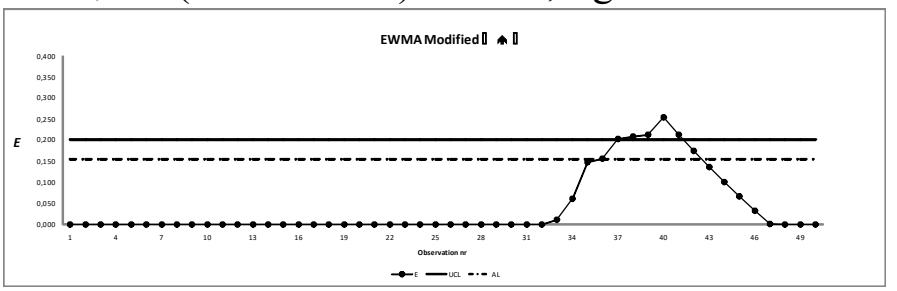

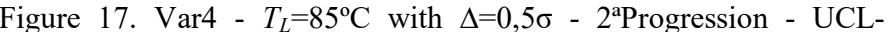
$\mathrm{ARL}=1000$



Figure 18. Var4 - $T_{L}=85^{\circ} \mathrm{C}$ with $\Delta=0,5 \sigma-3^{\text {aaProgression - UCL- }}$ $\mathrm{ARL}=1000$

For higher ARL values, the control chart is less sensible. Assuming navigation with higher sea temperatures, the ARL limit can be higher; for lower 
temperatures ARL can be lower, always respecting the manufacturer limits.

\section{CONCLUSIONS}

For auto correlated data, an ARIMA model can be used for data modeling. For independent data, observations are used directly for control charts equipment monitoring.

The autocorrelation model is chosen comparing the ACF and PACF with the EACF and EPACF.

In phase 1 the individual observations and moving range control charts are used to define the working parameters values. The parameters are defined after charts correction for outliers.

It's possible to monitoring equipment data with the Modified EWMA control charts.

The Modified EWMA is more sensible than the traditional charts.

The variability of $\Delta$ influences the charts sensitivity.

For higher ARL values EWMA the chart sensitivity decreases.

The analyzed equipment has shown parameters under statistical control.

\section{RECOMENDATIONS}

The simultaneous use of several techniques for equipment condition diagnostic is recommended.

Test the four variables data with MEWMA Charts, and use flexibility concept in the charts design.

Development of software to allow flexibility in the limits of online monitoring with control charts is needed.

\section{REFERENCES}

Rawat, Manish, Lad, Bhupesh (2013). Condition Based Optimal Maintenance Strategy for Multi-Component System. http://www.researchgate.net/publication/25844583, 20Jan2016.

Lampreia, Suzana (2013). Manutenção Baseada no Estado de Condição. Uma Abordagem Utilizando Cartas de Controlo Modificadas. Tese de Doutoramento, Faculdade de Ciências e Tecnologia da Universidade Nova de Lisboa, Setembro.

Schaeffers, Marc (2016). Manage control limits when Implementing Statistical Process Control. http://www.isixsigma.com/tools-templates/controlcharts/manage-control-limits-when-implem- enting-statistical-process-control 19Jan2016.

Pereira, Z. L. and Requeijo, J. G. (2012), Qualidade: Planeamento e Controlo Estatístico de Processos (Quality: Statistical Process Control and Planning), FCT/UNL Foundation Editor, Lisboa.

Lampreia, S., Barbosa, P., \& Requeijo, J. (2012). Manutenção Condicionada Baseada na Aplicação de Cartas Controlo EWMA. Congresso Ibérico de Jovens Engenheiros CIJE2012. Braga.

Lampreia, Suzana, Requeijo, José, Dias, José, Vairinhos, Valter (2013). Equipment Condition Monitoring with an Application of MEWMA Control Charts and Others Charts. ICOVP2013, Setembro de 2013, Lisboa.

Crowder, S. V., \& Hamilton, M. D. (1992). An EWMA for monitoring a process standard Deviation,Journal of Quality Technology, Vol. 24, pp. 12-21.

Zou, C., \& Tsung, F. (2010). Likelihood ratio-based distribution-free EWMA control charts. Journal of Quality Technology, pp. 174-196.

Serel, D. A., \& Moskowitz, H. (2008). Joint economicdesign of EWMA control charts for mean and variance, European Journal of Operational Research, pp. 157-168.

Crowder, S.V., A Simple Method for Studying Run Length Distributions of Exponentially Weighted Moving Average, Technometrics, Vol. 29, 1989, pp. 155-162. 